\title{
FIXED-POINT ANALYSIS OF MOBILENETS ON KEYWORD SPOTTING
}

\author{
Andrei-Marius AVRAM \\ Military Technical Academy, Bucharest, Romania \\ avram.andreimarius@gmail.com \\ Mihai COCA \\ Military Technical Academy, Bucharest, Romania \\ mihai.coca@mta.ro \\ Ștefan-Ciprian ARSENI \\ Military Technical Academy, Bucharest, Romania \\ stefan.arseni@mta.ro
}

\begin{abstract}
Automatic recognition of speech is a typical set of rules in the world of machine learning (ML). Due to the Internet of Things (IoT) markets forecasts, ML applications are moving from the Cloud to the nodes. Considering the need for near real-time response in IoT systems, one of the before mentioned ML applications is the Keyword Spotting (KWS) system, an intelligent assistant which can recognize different keywords pronounced by a user. These systems are now implemented on embedded devices which imply some limitations in terms of memory and computational power. Due to these limitations, quantifying the weights of a neural network, used by a KWS system, has become an important area of research. This paper analyses the possibility of diminishing the processing latency with respect to the amount of memory used by a lightweight neuronal network, through the quantization of its weights in fixed-point representation. The quantization is routed to the network at different levels, from 12 to 28 bits, gaining 20-bit accuracy stabilization. As a result, the memory footprint for 20bit quantization level is reduced by $37 \%$ than the standard floating-point representation of the weights, while the loss in accuracy is only $0.004 \%$.
\end{abstract}

Keywords: Depth-wise Separable Convolutional Neural Networks (DS-CNN), Fixed-Point, Keyword Spotting (KWS), MobileNets, Speech Recognition.

JEL classification: $\mathrm{C} 45$

DOI: $10.12948 / \mathrm{ie} 2019.01 .02$

\section{Introduction}

Speech recognition is the inter-disciplinary sub-field of computational linguistics that develops methodologies and technologies enabling computers to recognize and translate the spoken language into text. Neural networks have a long history in speech recognition, usually in combination with hidden Markov models. They have gained attention in recent years with the dramatic improvements in acoustic modelling yielded by deep recurrent neural networks [1]. These improvements also enabled them to surpass human accuracies in a variety of cognitive tasks, such as conversational speech recognition [2]. Considering this, speech is increasingly becoming a more natural way to interact with consumer electronic devices, for example, Amazon Echo and Google Home. One component of the system enabling the interaction between users and devices, is the Keyword Spotting. This component detects predefined keywords, similar to how an intelligent personal assistant does it (e.g. "Alexa" for Amazon Echo, "Ok Google" for Google Home or "Siri" for Apple HomePod), and wakes up the device, 
activating the full scale speech recognition either on device or in the Cloud. Since a KWS system is always-on, it should have very low power consumption to maximize battery life. On the other hand, the KWS system should detect the keywords with high accuracy and low latency, for best user experience. Microcontrollers are a great platform to deploy KWS implementations given their low-cost energy-efficient processors, which are becoming indispensable in our daily activities. However, microcontrollers come with significant memory and computational constraints. Typical microcontroller systems have only tens to few hundred KBs of memory available and, since the KWS system is always-on, the real-time requirement limits the total number of operations per neural network inference [3]. Taking into consideration these constraints of microcontrollers, the goal is to build a system that has low memory requirements and low latency, while providing a high accuracy in keyword spotting. To achieve these requirements, we propose a lightweight neural network and we analyse its memory requirements and accuracy in 32 floating-point and various fixed-point computations, that can be further utilized to choose an optimal model for the desired platform. We decided to use MobileNets [4] as the KWS model in our proposed architecture, given their use of depthwise separable convolutions for resource-efficient computations. The rest of the paper is organized as follows. Section 2 describes the proposed neural network architecture, the dataset and the training process, while Section 3 presents some details about the fixed-point implementation and the optimizations that have been done to further improve the performance of the model. Section 4 contains the experimental results of our analysis and a comparison with some recent deep learning fixed-point implementations. We conclude the paper with some points on future work and research directions.

\section{Neural Network Architecture}

Recently, depth-wise separable convolution has been proposed as an efficient alternative to the standard 3-D convolution operation [5] and has been used to achieve compact network architectures in the area of computer vision. DS-CNN (Depth-wise Separable Convolutional Neural Networks) first convolves each channel in the input feature map with a separate 2-D filter and then uses point-wise convolutions (e.g. 1x1) to combine the outputs in the depth dimension. By decomposing the standard 3-D convolutions into 2-D convolutions followed by 1-D convolutions, depth-wise separable convolutions are more efficient, both in number of parameters and operations, which makes deeper and wider architectures possible even in the resource-constrained microcontroller devices. Figure 1 shows how a standard convolution filter can be decomposed in a depth-wise and point-wise convolution filter.

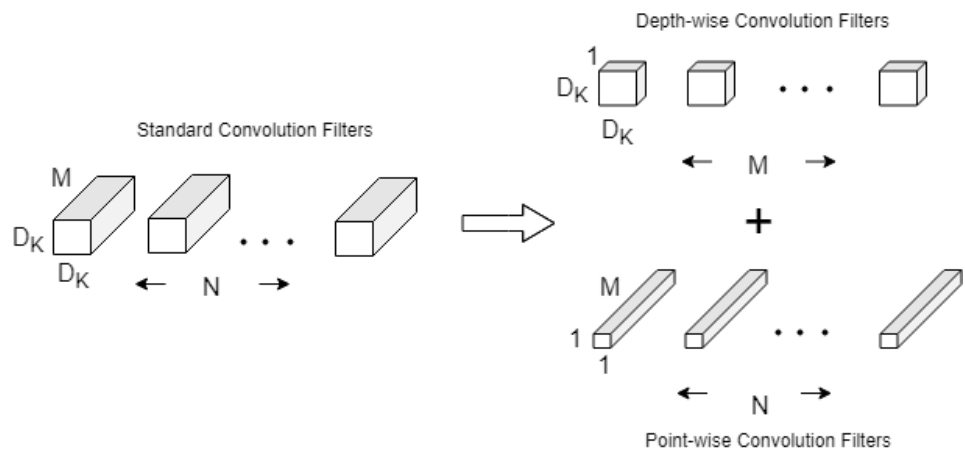

Figure 1. Decomposition of a standard convolution filter in a dept-wise separable and point-wise separable convolution filter

The convolutional layers in our architecture are used to process the Mel Frequency Cepstral Coefficients (MFCCs) of the signal, encoded in a 49x10 matrix. MFCCs are a commonly used 
feature of human-engineered speech in speech-recognition based on deep learning. This technique involves translating the time-domain speech signal into a set of frequency-domain spectral coefficients, which enables dimensionality compression of the input signal. The output of the convolution process is then fed in a fully-connected neural network that will classify the signal to a specific command. The architecture of our proposed model is further described in Figure 2.

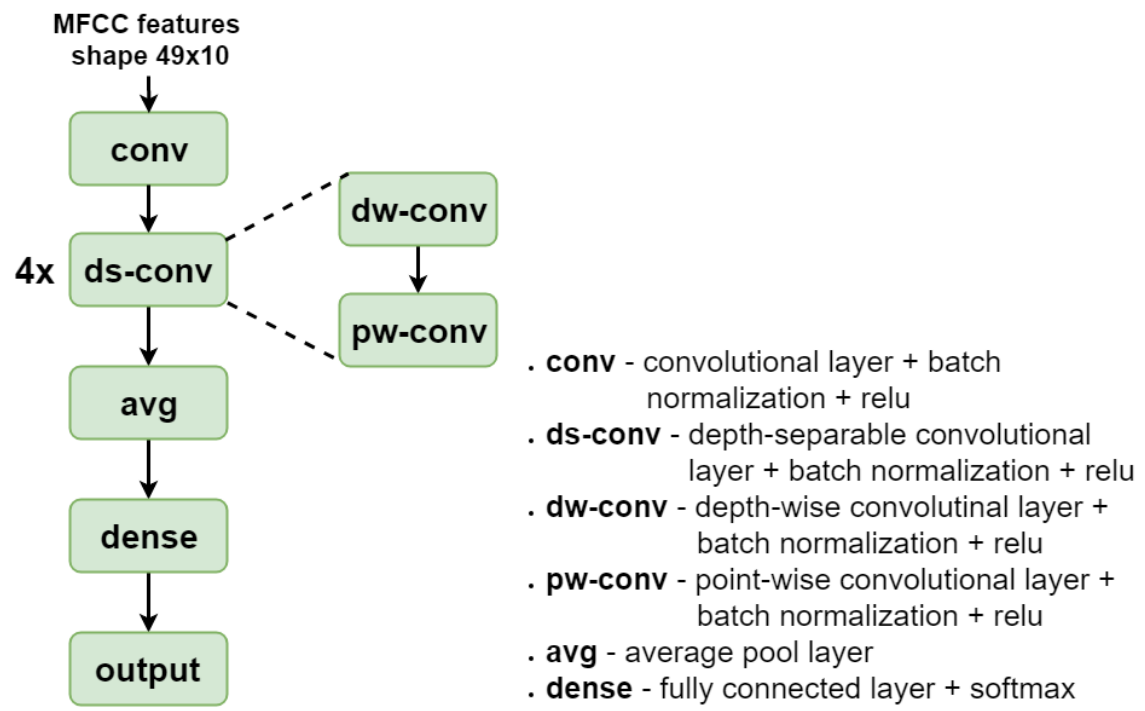

Figure 2. Architecture of our proposed model

The first layer of the neural network is a simple convolutional layer with 64 filters of size 10x4, padding "same" and a stripping of 2 on horizontal and vertical axes. The next four layers are the depth-separable convolutions that are used to speed-up the computations. They have 64 filters with kernel size of 3, padding "same" and a stripping of 1. After each convolutional layer, a relu activation function and a batch-normalization (to reduce the internal covariance shift) are used. The output of the final convolution is averaged across last dimension by using an average pool. The last layer of the neural network is a fully-connected layer with twelve neurons that, together with the softmax activation function, create the output of the model. As a result, the output of the model consists of a vector with values between 0 and 1, representing the probabilities of each command at the given index.

To create this architecture, we have chosen Google Tensorflow [6] framework because it provides a variety of different toolkits, enabling us to construct models at our preferred level of abstraction. We can use lower-level APIs to build models by defining a series of mathematical operations or we can use higher-level APIs (e.g. tf.keras) to specify predefined architectures, such as convolutional layers or dense layers.

The training has been done using the Google speech commands dataset [7] that is split into training, validation and test sets, in the ratio of 80:10:10. The dataset consists of 65.0001 second long audio clips of 30 keywords pronounced by thousands of different people. Each clip consists of only one of the 30 keywords. The neural network is trained to classify the incoming audio into one of the following 10 keywords: "Yes", "No", "Up", "Down", "Left", "Right", "On", "Off", "Stop", "Go". Along these mentioned keywords there are two more: "silence" (i.e. no word spoken) and "unknown" (which marks the remaining 20 keywords from the dataset). We used the standard cross-entropy and Adam optimizer, a batch size of 128 and a learning rate of 0.05 . The model has been trained for a total of 100 epochs. 
www.conferenceie.ase.ro

\section{Fixed-Point Analysis}

Neural networks are typically computational and memory expensive and many microcontrollers may be overwhelmed when trying to run them. This paper proposes a method of improving the speed of a neural network, by quantizing its weights in fixed-point numbers. Previous research $[8,9]$ has shown that neural networks can run in fixed-point with a minimal loss in accuracy. Moreover, the memory requirement to store the weights of the model is also lower. In our analysis we have taken the previously described neural network and quantized its weights to fixed-point numbers, represented in formats ranging from 12 bits to 28 bits. Let $L$ be the number of bits that represent the difference between 32 and the total number of bits that we choose to represent the value and $F$ be the number of bits that represent the fractional part. To make the conversion, we shift to the left the floating-point value by $L+F$ bits and take the nearest 32-bit integer value of the obtained number. The reverse conversion was done by shifting the integer value to the right by $L+F$ bits and storing the resulting number in float representation.

One of the difficulties that we encountered was that the dense layer activation function is a softmax function, presented in (1), and we needed to compute the value of $e^{z}$ in fixed-point.

$$
\sigma(z)_{j}=\frac{e^{z_{j}}}{\sum_{k=1}^{K} e^{z_{k}}}, j=\overline{1, K}, z=\left(z_{1}, \ldots, z_{K}\right) \in R^{K}
$$

We could have used various algorithms as Taylor Series to approximate it, but, because the model is already trained when it is deployed to the platform, we can safely remove the activation function at inference time. The reason behind this is that the softmax function does not affect the index of the command with the highest value. If we consider $x$ to be the output of the dense layer, then we can affirm that (2) is true, because the softmax function only amplifies the values in a set through exponentiation and brings the values in $[0,1]$ interval through normalization.

$$
\underset{x}{\operatorname{argmax}} x=\underset{x}{\operatorname{argmax}} \operatorname{sofmax}(x)
$$

Moreover, by removing it, there will be less computation overhead at each prediction, thus improving the near real-time requirements of the system.

\section{Testing and Analysis}

The KWS is an active research field and many models have been proposed, with different tradeoffs between performance, precision and memory consumption. For instance, in [10] authors implement a convolutional recurrent neural network to detect the keyword "TalkType" (which can be pronounced as a single word or two words) and they obtained a model with $97.71 \%$ accuracy. Usually, KWS systems are implemented on limited memory embedded devices generating an empowered reason for weights quantization. In [8], authors use floating-point numbers for representing the weights and fixed-point numbers for representing the activation functions on popular large-scale CNNs, such as AlexNet, SqueezeNet, GoogLeNet and VGG16. Their experimental results show that the proposed scheme reduces the weight storage by up to $36 \%$ and power consumption of the hardware multiplier by up to $50 \%$.

In our research, we aimed to obtain both a high performance for the KWS task and a high memory reduction of the weight storage from floating-point to fixed-point. The evaluation of our DS-CNN was done on the test set, that represent $10 \%$ of the Google speech commands dataset. As depicted in Table 1, the neural network has obtained an average accuracy of $95.84 \%$ on 32 bits floating-point and an exponential increasing average accuracy from $0.02 \%$, on 12 bits fixed-point, to $95.54 \%$, on 28 bits fixed-point. This increase is the result of the reduction of the overflow and underflow errors that are propagated throughout the model. Based on these 
results, we can observe that the optimal quantization would be on 20 bits, because the difference between the accuracies obtained by adding more bits is negligible ( 24 bits or 28 bits). In terms of memory consumption, it can be observed in Table 1 that we need 62,3 KBs to store the model on 20 bits fixed-point (the number of bits of optimal accuracy) compared to $99,6 \mathrm{KBs}$ on 32 bits floating-point. This is a reduction of more than $37 \%$ and this memory requirement would be sufficient for most embedded systems.

Table 1. Accuracy of the proposed model on floating- and fixed-point

\begin{tabular}{|c|c|c|c|c|c|c|}
\hline Command & $\begin{array}{l}32 \text { bits } \\
\text { floating- } \\
\text { point }\end{array}$ & $\begin{array}{l}12 \text { bits } \\
\text { fixed- } \\
\text { point }\end{array}$ & $\begin{array}{l}16 \text { bits } \\
\text { fixed- } \\
\text { point }\end{array}$ & $\begin{array}{l}20 \text { bits } \\
\text { fixed- } \\
\text { point }\end{array}$ & $\begin{array}{l}24 \text { bits } \\
\text { fixed- } \\
\text { point }\end{array}$ & $\begin{array}{l}28 \text { bits } \\
\text { fixed- } \\
\text { point }\end{array}$ \\
\hline DOWN & 0.953 & 0.008 & 0.136 & 0.953 & 0.953 & 0.953 \\
\hline GO & 0.971 & 0.013 & 0.290 & 0.962 & 0.962 & 0.965 \\
\hline LEFT & 0.962 & 0.017 & 0.415 & 0.953 & 0.953 & 0.955 \\
\hline NO & 0.962 & 0.021 & 0.227 & 0.954 & 0.954 & 0.954 \\
\hline OFF & 0.936 & 0.008 & 0.258 & 0.932 & 0.932 & 0.932 \\
\hline ON & 0.945 & 0.030 & 0.342 & 0.941 & 0.941 & 0.941 \\
\hline RIGHT & 0.958 & 0.008 & 0.342 & 0.949 & 0.949 & 0.949 \\
\hline SILENCE & 0.981 & 0.032 & 0.333 & 0.978 & 1.000 & 1.000 \\
\hline STOP & 0.945 & 0.055 & 0.324 & 0.950 & 0.950 & 0.95 \\
\hline UNKNOWN & 0.968 & 0.032 & 0.176 & 0.960 & 0.960 & 0.96 \\
\hline UP & 0.941 & 0.029 & 0.555 & 0.937 & 0.937 & 0.937 \\
\hline YES & 0.979 & 0.017 & 0.286 & 0.979 & 0.979 & 0.979 \\
\hline AVERAGE & 0.9584 & 0.0225 & 0.3070 & 0.9540 & 0.9555 & 0.9562 \\
\hline MEMORY & 99.6KB & $37.3 \mathrm{~KB}$ & $49.8 \mathrm{~KB}$ & 62.3KB & 74.7KB & 87.5KB \\
\hline
\end{tabular}

Accuracy can be a misleading measurement in classification tasks that are done on skewed datasets [11]. Therefore, we have also calculated the precision and the recall for the 20 bits fixed-point optimal model to gain more insight about its performance, obtaining the scores of $90.14 \%$ and $95.55 \%$, respectively. The high scores of both precision and recall show that the model is not biased towards some class due to data imbalance.

\section{Conclusions and future work}

Hardware optimized neural network architectures are key to get efficient results on resource constrained microcontrollers. Our proposed model of DS-CNN architecture was implemented and trained to recognize 12 keywords from the Google speech commands dataset and, during tests it obtained an accuracy of $95.84 \%$. To further increase the speed of the computations, we quantized the weights of the neural network in various fixed-point representations and observed that for representations greater than 20 bits, the accuracy loss would become negligible. Moreover, the quantization would have the benefit of reducing the memory requirements of the neural network with more than $37 \%$. As future research directions of this work, we will continue with the implementation of the neural network on a field-programmable gate array (FPGA), while analysing the near real-time performance of the system. The code and the pretrained models are available at [12]. 
www.conferenceie.ase.ro

\section{Acknowledgment}

This work was supported by a grant of the Romanian Ministry of Research and Innovation, CCCDI - UEFISCDI, project number PN-III-P1-1.2-PCCDI-2017-0272/Avant-garde Technology Hub for Advanced Security (ATLAS), within PNCDI III.

\section{References}

[1] A. Graves, A.-r. Mohamed and G. Hinton, "Speech recognition with deep recurrent neural networks," in 2013 IEEE international conference on acoustics, speech and signal processing, IEEE, 2013, pp. 6645-6649.

[2] W. Xiong, L. Wu, F. Alleva, J. Droppo, X. Huang and A. Stolcke, "The Microsoft 2017 conversational speech recognition system," in 2018 IEEE International Conference on Acoustics, Speech and Signal Processing (ICASSP), IEEE, 2018, pp. 5934-5938.

[3] X. Xu, Y. Ding, S. X. Hu, M. Niemier, J. Cong, Y. Hu and Y. Shi, "Scaling for edge inference of deep neural networks," Nature Electronics, vol. 1, p. 216, 2018.

[4] A. G. Howard, M. Zhu, B. Chen, D. Kalenichenko and W. Wang, "Mobilenets: Efficient convolutional neural networks for mobile vision applications," arXiv preprint arXiv:1704.04861, 2017.

[5] F. Chollet, "Xception: Deep learning with depthwise separable convolutions," in Proceedings of the IEEE conference on computer vision and pattern recognition, 2017, pp. 1251-1258.

[6] M. Abadi, P. Barham, J. Chen, Z. Chen, A. Davis, J. Dean and M. Devin, "Tensorflow: A system for large-scale machine learning," Symposium on Operating Systems Design and Implementation, pp. 265-283, 2016.

[7] P. Warden, "Speech Commands: A public dataset for single-word speech recognition," Dataset available from http://download. tensorflow. org/data/speech $\_$commands $\backslash$ v0, vol. $1,2017$.

[8] L. Lai, N. Suda and V. Chandra, "Deep convolutional neural network inference with floating-point weights and fixed-point activations," arXiv preprint arXiv:1703.03073, 2017.

[9] J. Qiu et al., "Going deeper with embedded fpga platform for convolutional neural network," in Proceedings of the 2016 ACM/SIGDA International Symposium on FieldProgrammable Gate Arrays, ACM, 2016, pp. 26-35.

[10] S. O. Arik, M. Kliegl, R. Child, J. Hestness, A. Gibiansky, C. Fougner, R. Prenger and A. Coates, "Convolutional recurrent neural networks for small-footprint keyword spotting," arXiv preprint arXiv:1703.05390, 2017.

[11] B. J. M. Abma, "Evaluation of requirements management tools with support for traceability-based change impact analysis.," Master's thesis, University of Twente, Enschede, 2009.

[12] A. M. Avram, "Fixed-Point Analysis of MobileNets on Keyword Spotting," [Online]. Available: https://github.com/avramus/MobileNet-fixedpoint-analysis. 